\title{
MicroRNA-142-5p modulates breast cancer cell proliferation and apoptosis by targeting phosphatase and tensin homolog
}

\author{
WENDA XU and WEIXING WANG \\ Department of General Surgery, Renmin Hospital of Wuhan University, Wuhan, Hubei 430060, P.R. China
}

Received June 15, 2017; Accepted January 12, 2018

DOI: $10.3892 / \mathrm{mmr} .2018 .8812$

\begin{abstract}
A total of 60 breast cancer (BC) tissues and adjacent healthy tissues from patients who underwent surgery in Renmin Hospital of Wuhan University were collected for analysis in the present study. Results from reverse transcription-quantitative polymerase chain reaction (RT-qPCR) demonstrated that, compared with the adjacent healthy tissues, the expression levels of microRNA (miR)-142-5p were significantly elevated in BC tissues. Bioinformatics analysis was performed using TargetScan for the prediction of potential target sites that matched the seed region of miR-142-5p; phosphatase and tensin homolog (PTEN) exhibited the highest score and was selected for further analysis. Results of RT-qPCR analysis demonstrated that, compared with the adjacent healthy tissues, the mRNA expression levels of PTEN were significantly decreased in breast cancer tissues. miR-142-5p and PTEN expression levels were positively and negatively associated, respectively, with patient tumor size and metastasis. MDA-MB-231 cells were divided into three groups including the Control group, the miR-NC inhibitor group and the miR-142-5p inhibitor group. As for alterations in cell behavior, including cell viability and cell apoptosis, and protein expression levels, there were no significant differences between Control and miR-NC inhibitor groups. MTT assay results revealed that, compared with Control and miR-NC inhibitor groups, miR-142-5p inhibitor reduced MDA-MB-231 cell proliferation. Flow cytometric analysis demonstrated that, compared with Control and miR-NC inhibitor groups, miR-142-5p inhibitor treatment induced MDA-MB-231 cell apoptosis. Western blotting results demonstrated that, compared with Control and miR-NC inhibitor groups, miR-142-5p inhibitor treatment significantly increased the expression of PTEN, reduced the activation of phosphatidylinositol-4,5-bisphosphate 3-kinase/R ACa
\end{abstract}

Correspondence to: Dr Weixing Wang, Department of General Surgery, Renmin Hospital of Wuhan University, 99 Zhang Zhidong Road, Wuhan, Hubei 430060, P.R. China

E-mail: wangweixingrmh@sina.com

Key words: microRNA-142-5p, breast cancer, phosphatase and tensin homolog serine/threonine-protein kinase signaling. Finally, PTEN was demonstrated to interact with miR-142-5p from the results of dual-luciferase reporter assay in the present study. The findings of the present study suggested that miR-142-5p may be a potential therapeutic target for the future investigations and insights for breast cancer.

\section{Introduction}

Breast cancer (BC) is the leading cause of cancer-related mortality in women between the ages of 20 and 59 years $(1,2)$, and affects $12 \%$ of women worldwide (3). A number of risk factors have been associated with the occurrence of $\mathrm{BC}$ in women, including obesity, early menarche, late menopause, hormone replacement therapy, late first-full pregnancy and diets rich in high-fat foods or red meats (4). Therefore, it is necessary to investigate effective diagnostic methods for $\mathrm{BC}(5)$.

MicroRNAs (miRNAs) are a class of small non-coding RNAs that provide a novel perspective to predict and screen for cancer, and previous studies have reported that miRNA expression levels may be aberrant in tumors (6-8). Associations between the clinicopathological features of $\mathrm{BC}$ and miRNA expression levels have been reported previously $(9,10)$. miR-142-3p was demonstrated to directly regulate its target genes in a several types of cancer, including non-small cell lung cancer, colon cancer and hepatic cancer (11-13). miR-142 expression levels were elevated in human BC stem cells (BCSCs) compared with non-tumorigenic BC cells (14). In addition, miR-142-5p expression levels were reported to be markedly higher in the lymph node-cancer group compared with expression in the non-lymph node-cancer group, which were detected by miRNA microarray analysis (15). Upregulation of miR-142-5p was identified in atherosclerotic plaques, and regulated oxidized low-density lipoprotein-induced apoptosis in macrophages (16). miR-142-5p was involved in squamous lung cancer via regulation of cell cycle related genes (17). miR-142-5p contributes to Hashimoto's thyroiditis by targeting CLDN1 (18) and promotes the development of colorectal cancer by targeting SDHB (19). However, the function of miR-142-5p in BC has not yet been reported. Consequently, the present study aimed to investigate a target gene of miR-142-5p in $\mathrm{BC}$ cells and to provide a novel potential target for the treatment of $\mathrm{BC}$. 


\section{Materials and methods}

Study population. BC tissues and the adjacent healthy tissues were obtained from 60 female patients who underwent surgery in Renmin Hospital of Wuhan University (Wuhan, China) between March, 2013 and July, 2016. Clinicopathological characteristics of patients' age, tumor size and metastasis were collected and are presented in Table I. There were 40 patients aged $<50$ years and 20 patients aged $\geq 50$ years. Written informed consent was obtained from all the patients prior to the start of the study; the present study was approved by the ethics committee of Renmin Hospital of Wuhan University.

Cell culture. Normal breast epithelial cell line MCF-10A and BC cell lines SK-BR-3 and MDA-MB-231 were purchased from the American Type Culture Collection (Manassas, VA, USA) and cultured in Dulbecco's modified Eagle's medium (DMEM)/Ham's F12 medium supplemented with 10\% fetal bovine serum (Gibco; Thermo Fisher Scientific, Inc., Waltham, MA, USA), penicillin $(100 \mathrm{U} / \mathrm{ml})$ and streptomycin $(100 \mu \mathrm{g} / \mathrm{ml})$ in an incubator at $37^{\circ} \mathrm{C}$ with humidified atmosphere of $5 \% \mathrm{CO}_{2}$. MDA-MB-231 cells with the highest miR-142-5p expression levels were selected for the remaining in vitro experiments as these cells exhibited the highest expression levels of miR-142-5p compared with other cell lines.

Reverse transcription-quantitative polymerase chain reaction $(R T-q P C R)$. Total RNA was extracted from BC tissues $\left(1 \mathrm{~cm}^{3}\right)$ and cell cultures $\left(1 \times 10^{6}\right.$ cells/well) using a miRNeasy kit (Qiagen Inc., Valencia, CA, USA) according to the manufacturer's protocol. Reverse transcription of miR142-5p and phosphatase and tensin homolog (PTEN) to cDNA was performed using miRNA cDNA Synthesis Kit (Takara bio, Inc., Otsu, Japan) and First Strand cDNA Synthesis kit (Takara, Dalian, China), respectively. Expression levels of miR-142-5p and PTEN were detected with a TaqMan miRNA assay kit (Applied Biosystems; Thermo Fisher Scientific, Inc.) on an ABI 7500 thermocycler (Applied Biosystems; Thermo Fisher Scientific, Inc.), according to the manufacturer's protocol. Thermocycling conditions were as following: $95^{\circ} \mathrm{C}$ for $30 \mathrm{sec}$, followed by 35 cycles of $95^{\circ} \mathrm{C}$ for $10 \mathrm{sec}$ and $60^{\circ} \mathrm{C}$ for $25 \mathrm{sec}$. Relative expression levels of miRNA-142-5p were normalized to U6, and relative PTEN expression levels were normalized to GAPDH. Primers were as follows: PTEN, forward 5'-TGGATT CGACTTAGACTTGACCT-3', reverse 5'-GGTGGGTTATGG TCTTCAAAAGG-3'; GAPDH, forward 5'-ACAAGATGG TGAAGGTCGGTGTGA-3', reverse 5'-AGCTTCCCATTC TCAGCCTTGACT-3'; miR-142-5p, forward 5'-AACTCCAGC TGGTCCTTAG-3', reverse 5'-TCTTGAACCCTCATCCTG T-3'; and U6, forward 5'-GCTTCGGCAGCACATATACTA AAAT-3', reverse 5'-CGCTTCACGAATTTGCGT-3'. The expression levels were compared with the $2^{-\Delta \Delta \mathrm{Cq}}$ method (20).

Plasmid transfection. MDA-MB-231 cells $\left(1 \times 10^{5}\right.$ cells/well) were seeded in 24-well plates and transfected with $30 \mu \mathrm{M}$ miR-142-5p inhibitor or miR-negative control (NC) inhibitor using Lipofectamine ${ }^{\circledR} 2000$ (Thermo Fisher Scientific, Inc.) and incubated at $37^{\circ} \mathrm{C}$ for $48 \mathrm{~h}$ according to the manufacturer's protocol. MDA-MB-231 cells were randomly divided into three groups, including the Control group (untreated cells), the miR-142-5p inhibitor (5'-AGUAGUGCUUUCUAC UUUAUG-3'; Guangzhou RiboBio Co., Ltd.) group and the miR-NC inhibitor (5'-CAGUACUUUUGUGUAGUACAA-3'; Guangzhou RiboBio Co., Ltd.) group. Cells were collected at $48 \mathrm{~h}$ after plasmid transfection for the following experiments.

Cell viability analysis. An MTT assay was conducted to evaluate cell viability. MDA-MB-231 cells from each of the three groups were seeded $\left(3 \times 10^{4}\right.$ cells/well $)$ in 96-well plates and incubated for 12, 24 and $48 \mathrm{~h}$. Following the addition of MTT $(5 \mathrm{mg} / \mathrm{ml})$ into each well, cells were incubated for $1.5 \mathrm{~h}$ at $37^{\circ} \mathrm{C}$. Subsequently, the supernatant was discarded, $200 \mu 1$ dimethylsulfoxide was added to dissolve the formazan crystals and the optical density was evaluated by reading the absorbance at $450 \mathrm{~nm}$ of each well with a spectrophotometer.

Cell apoptosis analysis. MDA-MB-231 cells $\left(2 \times 10^{5}\right.$ cells/well) were seeded in 12-well plates and cultured for $48 \mathrm{~h}$ in an incubator at $37^{\circ} \mathrm{C}$ in a humidified atmosphere of $5 \% \mathrm{CO}_{2}$. The cells were collected by centrifugation at of $23,200 \mathrm{x} \mathrm{g}$ at $4{ }^{\circ} \mathrm{C}$ for $5 \mathrm{~min}$, washed with cold PBS and fixed in ice-cold $70 \%$ ethanol overnight at $-20^{\circ} \mathrm{C}$. Cells were subsequently stained with annexin V-fluorescein isothiocyanate and propidium iodide (Roche Diagnostics, Basel, Switzerland) for $15 \mathrm{~min}$ at room temperature in the dark. Cells were collected and the percentage of cells with apoptotic nuclei (early and late apoptosis) was calculated using a flow cytometer (Beckman Coulter, Inc., Miami, FL, USA) and analyzed by Cell Quest software version FCS2.0 (BD Biosciences, Franklin Lakes, NJ, USA).

Western blotting. Total protein was extracted from MDA-MB-231 cell ( $1 \times 10^{5}$ cells/plate of 6-well plates) using radioimmunoprecipitation assay buffer (Beyotime Institute of Biotechnology, Shanghai, China). Protein concentration was determined by a bicinchoninic acid kit (Beyotime Institute of Biotechnology). Proteins (15 $\mu \mathrm{g} / \mathrm{lane})$ were separated by $10 \%$ SDS-PAGE and transferred onto polyvinylidene difluoride membranes. The membranes were blocked with $5 \%$ non-fat milk at room temperature for $2 \mathrm{~h}$ and incubated with the following primary antibodies overnight at $4{ }^{\circ} \mathrm{C}$ : PTEN $(1: 1,000$; cat. no. 9552; Cell Signaling Technology, Inc., Danvers, MA, USA); phosphorylated (p)-phosphoinositide 3-kinase (PI3K; 1:1,000; cat. no. 4228; Cell Signaling Technology, Inc.); p-RAC $\alpha$ serine/threonine-protein kinase (AKT; 1:1,000; cat. no. D9E; Cell Signaling Technology, Inc.); and GAPDH (1:1,000; cat. no. FL-355; Santa Cruz Biotechnology, Inc., Dallas, TX USA). Subsequently, membranes were incubated with anti-rabbit horseradish peroxidase-conjugated secondary antibody (1:1,000; cat. no. 7074; Cell Signaling Technology, Inc.) for $2 \mathrm{~h}$ at room temperature. Following washing with TBS $+0.1 \%$ Tween-20, blots were visualized with an Enhanced Chemiluminescence kit (Beyotime Institute of Biotechnology). Experiments were repeated in triplicate. Protein expression levels were normalised to GAPDH, and Quantity One version 4.6.2 (Bio-Rad Laboratories, Inc., Hercules, CA, USA) was used for densitometric analysis.

Luciferase activity assay. To verify whether PTEN was a direct target of miR-142-5p, a position of the PTEN 3'UTR containing a putative miR-142-5p target site was cloned into the luciferase 
Table I. Association between miR-142-5p or PTEN and patients clinicopathological characteristics.

\begin{tabular}{|c|c|c|c|c|c|}
\hline Factor & $\mathrm{n}$ & $\operatorname{miR}-142-5 p^{a}$ & P-value & PTEN $^{\mathrm{a}}$ & P-value \\
\hline Age (years) & & & 0.92 & & 0.73 \\
\hline$<50$ & 40 & $2.33 \pm 0.59$ & & $0.69 \pm 0.21$ & \\
\hline$\geq 50$ & 20 & $2.42 \pm 0.45$ & & $0.58 \pm 0.13$ & \\
\hline Tumor size $(\mathrm{cm})$ & & & $0.04^{\mathrm{b}}$ & & $0.01^{\mathrm{b}}$ \\
\hline$\geq 5$ & 18 & $2.67 \pm 0.28$ & & $0.37 \pm 0.22$ & \\
\hline$<5$ & 42 & $2.09 \pm 0.14$ & & $0.96 \pm 0.12$ & \\
\hline Metastasis & & & $0.04^{\mathrm{b}}$ & & $0.01^{\mathrm{b}}$ \\
\hline No & 32 & $2.25 \pm 0.21$ & & $0.96 \pm 0.24$ & \\
\hline Yes & 28 & $2.53 \pm 0.12$ & & $0.32 \pm 0.11$ & \\
\hline
\end{tabular}

${ }^{\mathrm{a} D a t a}$ are presented as the mean \pm standard error of the mean of expression levels in $\mathrm{BC}$ tissue compared with adjacent healthy tissue. ${ }^{\mathrm{b}} \mathrm{P}<0.05$. miR, microRNA; PTEN, phosphatase and tensin homolog.

open reading frame. The plasmids of PTEN were cloned into reporter pLuc control vector (Promega Corporation, Madison, WI, USA). MDA-MB-231 cells ( $1 \times 10^{4}$ cells/plate) were seeded in 48 -well plates and incubated for $24 \mathrm{~h}$ in an incubator at $37^{\circ} \mathrm{C}$ in a humidified atmosphere of $5 \% \mathrm{CO}_{2}$, followed by co-transfection with miR-142-5p inhibitor (20 nM) or miR-NC inhibitor (20 nM) and either PTEN wild-type (WT)-3' untranslated region (UTR; $1 \mathrm{mg}$, pLuc-PTEN-WT 3'UTR) or PTEN mutant (Mut)-3'UTR (1 mg, pLuc-PTEN-Mut 3'UTR) by Lipofectamine ${ }^{\circledR} 2000$ (Thermo Fisher Scientific, Inc.). After $48 \mathrm{~h}$ transfection, the luciferase activity was examined by Dual Luciferase Reporter Assay system (Promega Corporation, Madison, WI, USA), and the results were normalized to the Renilla luciferase activity.

Statistical analysis. Data were analyzed by software of SPSS version 21.0 (IBM Corp., Armonk, NY, USA) and expressed as the mean \pm standard error of mean. Data between two groups were analyzed by t-test and data among three groups were analyzed by one-way analysis of variance followed with Student-Newman-Keuls test. $\mathrm{P}<0.05$ was considered to indicate a statistically significance difference.

\section{Results}

Differing miR-142-5p expression levels between tumoral tissues and adjacent healthy tissues. RT-qPCR was used to analyze the relative expression levels of miR-142-5p in tumoral tissues and adjacent healthy tissues. The results demonstrated that miR-142-5p expression levels were significantly higher in the BC tissues compared with the adjacent healthy tissues (Fig. 1). This result suggested that miR-142-5p may be an oncogene during the development of $\mathrm{BC}$.

PTEN may be a target of miR-142. Potential targets of miR-142-5p were predicted by TargetScan (http://www .targetscan.org/vert_71), and the analysis revealed three target sequences of miR-142-5p in positions 2,189-2,195, 2,427-2,433 and 2,503-2,509 within the 3'UTR of PTEN (data not shown). The target site at position 2,189-2,195 scored 59 (the lowest), the

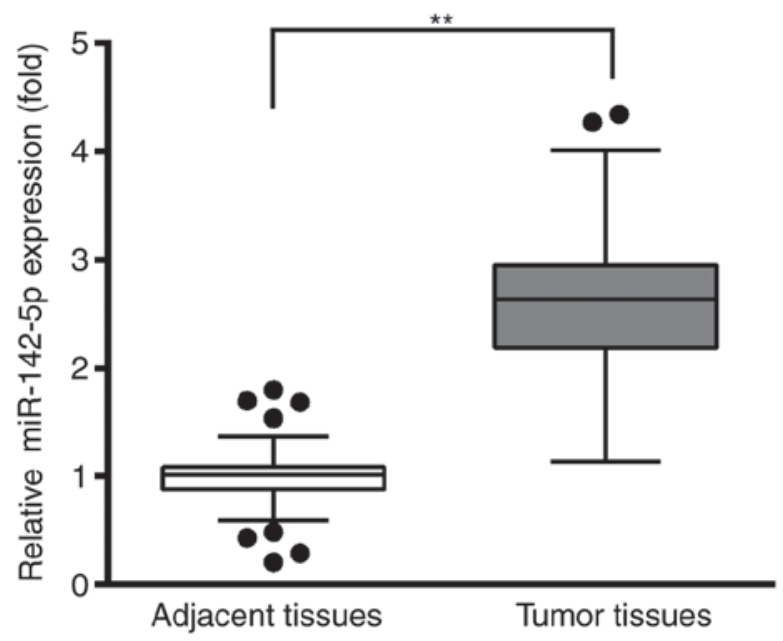

Figure 1. miR-142-5p expression levels are upregulated in BC tissues. Reverse transcription-quantitative polymerase chain reaction demonstrated that miR-142-5p expression levels were higher in BC tissues compared with expression levels in adjacent healthy tissues. ${ }^{* *} \mathrm{P}<0.01$. BC, breast cancer; miR, microRNA.

target sequence at position 2,427-2,433 scored 88 and at position 2,503-2,509 scored 89, which was highest (Fig. 2A). Therefore, position 2,503-2,509 of 3'UTR of PTEN was used for further analysis. RT-qPCR was used to examine the relative expression levels of PTEN in BC tissues and the adjacent healthy tissues. The results demonstrated that PTEN mRNA expression levels were lower in BC tissues compared with adjacent healthy tissues (Fig. 2B). This result suggested that PTEN may be a tumor suppressor gene during the development of BC.

Association between miR-142-5p or PTEN expression and patient clinicopathological characteristics. The characteristics of 60 patients with BC including age, tumor size and metastasis were presented in Table I. Gene expression levels of miR-142-5p and PTEN in BC tissues were investigated using RT-qPCR; results were presented as fold change compared with the adjacent healthy tissues. No significant associations were identified between miR-142-5p or PTEN expression level 

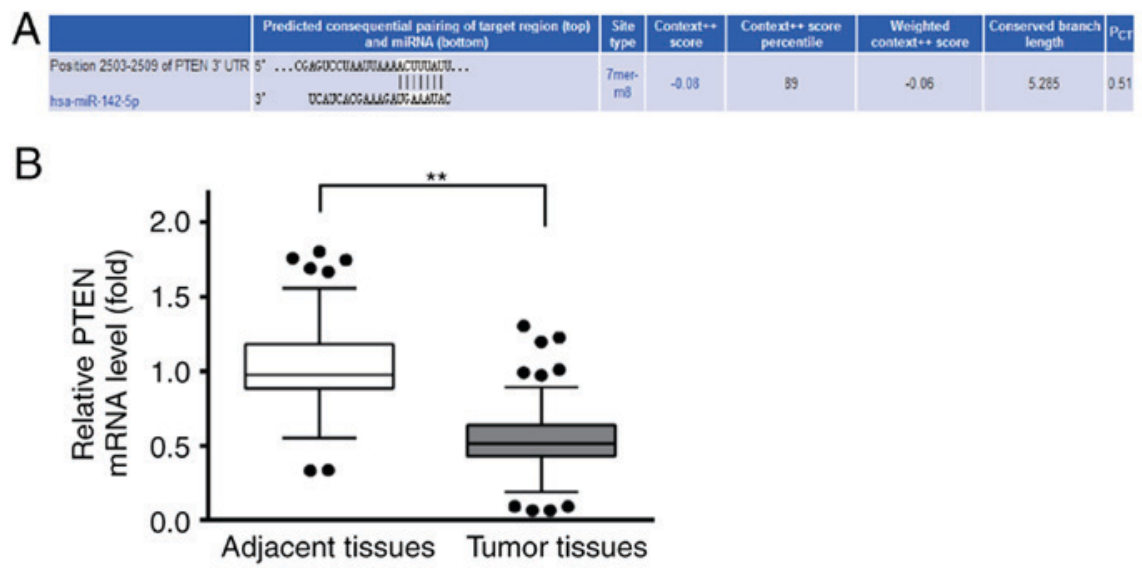

Figure 2. miR-142-4p may target PTEN. (A) miR-142-5p was predicted to target PTEN 3'UTR. (B) Lower expression levels of PTEN were demonstrated by reverse transcription-quantitative polymerase chain reaction in breast cancer tissues compared with expression in the adjacent healthy tissues. ${ }^{* *} \mathrm{P}<0.01$. miR, microRNA; PTEN, phosphatase and tensin homolog; UTR, untranslated region.
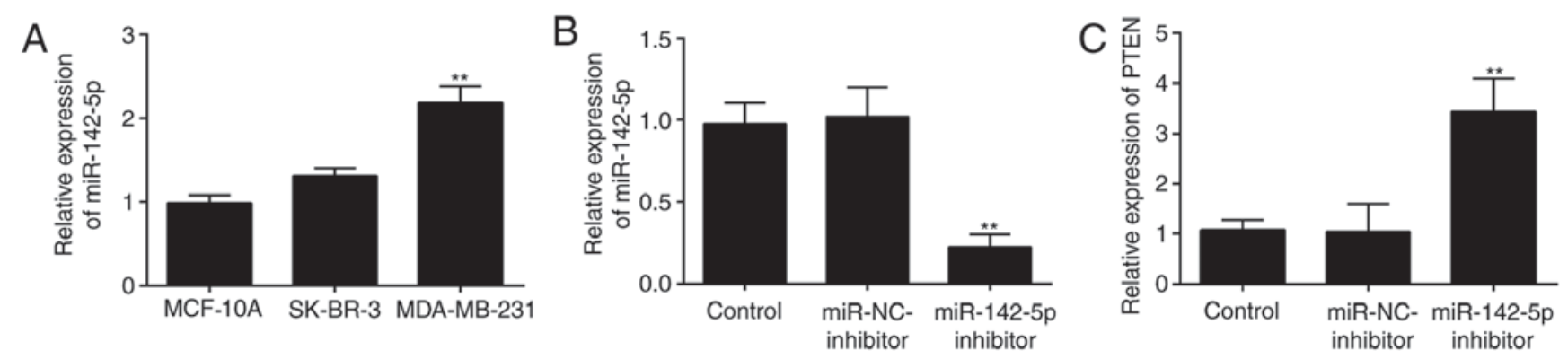

Figure 3. miR-142-5p expression levels BC cell lines. (A) Reverse transcription-quantitative polymerase chain reaction results indicated that MDA-MB-231 BC cells expressed the highest levels of miR-142-5p. ${ }^{* *} \mathrm{P}<0.01$ vs. MCF-10A or SK-BR-3 cells. (B and C) MDA-MB-231 cells were used in subsequent experiments, which demonstrated that (B) miR-142-5p expression was reduced in cells transfected with the miR-142-5p inhibitor, and (C) PTEN expression levels were increased in cells transfected with the miR-142-5p inhibitor. ${ }^{* *} \mathrm{P}<0.01$ vs. control or NC group. miR, microRNA; PTEN, phosphatase and tensin homolog; NC, negative control.

and patient age. Conversely, compared with tumor size $<5 \mathrm{~cm}$ and patients with no metastasis, miR-142-5p expression levels were significantly higher $(\mathrm{P}=0.04)$, whereas $\mathrm{PTEN}$ expression levels were significantly lower $(\mathrm{P}=0.01)$ in patients with tumors $\geq 5 \mathrm{~cm}$ and who exhibited metastasis.

Relative expression levels of miR-142-5p in MCF-10A, $S K-B R-3$ and MDA-MB-231 cell lines. RT-qPCR analysis was performed to investigate the variations in miR-142-5p expression levels among three different cell lines. The results indicated that miR-142-5p expression levels were the lowest in MCF-10A normal breast epithelial cells, no significant difference was observed between MCF-10A and SK-BR-3, whereas the highest expression level was detected in MDA-MB-231 BC cells (Fig. 3A). Therefore, MDA-MB-231 cells were selected for further analysis in the present study. The MDA-MB-231 cells were divided into three groups, including the Control group, the miR-NC inhibitor group and the miR-142-5p inhibitor group. The successful transfection of miR-142-5p inhibitor into MDA-MB-231 cells was identified by the reduced miR-142-5p expression level in the miR-142-5p inhibitor-treated group compared with the other two groups (Fig. 3B).

In addition, the effects of miR-142-5p inhibition on PTEN expression were investigated by RT-qPCR. In MDA-MB-231

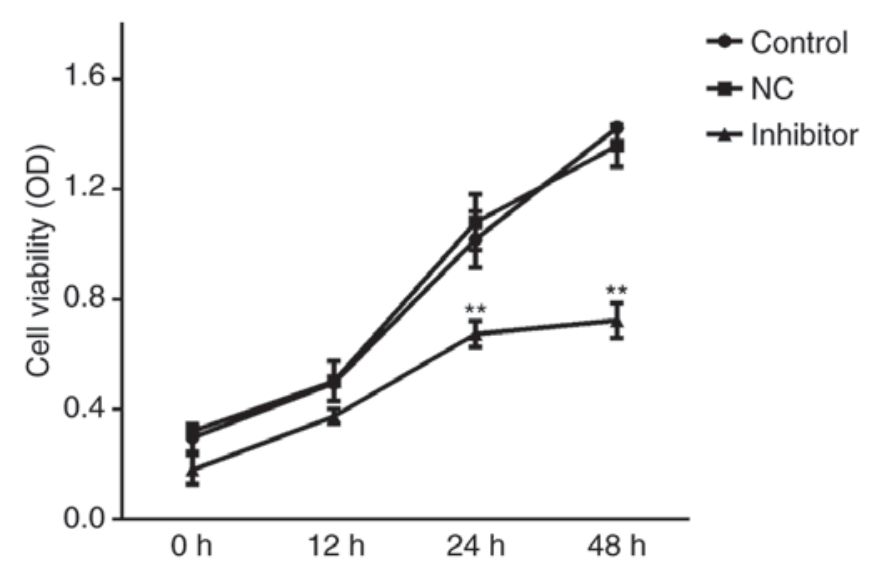

Figure 4. Inhibition of miR-142-5p reduces MDA-MB-231 cell viability. MTT assay results revealed that, there was no significant difference between the control and NC groups, and MDA-MB-231 cell viability was reduced by the inhibition of miR-142-5p at 24 and $48 \mathrm{~h}$ following transfection compared with in the control and NC groups. ${ }^{* *} \mathrm{P}<0.01$ vs. control or NC group. miR, microRNA; NC, negative control.

cells transfected with miR-142-5p inhibitor, the mRNA expression levels of PTEN were significantly increased compared with Control group and miR-NC group (Fig. 3C). These results 
A
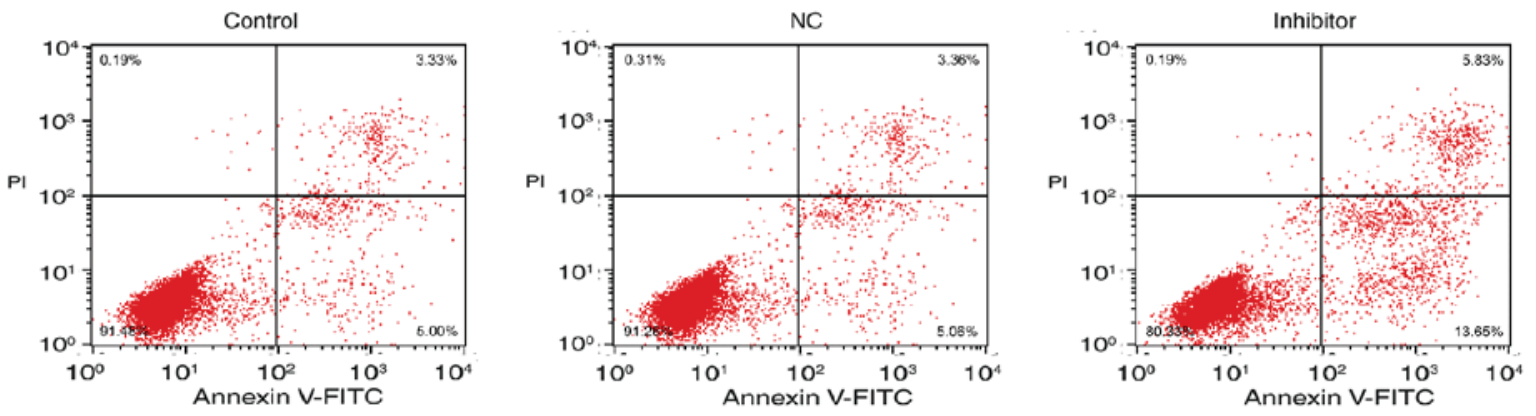

B

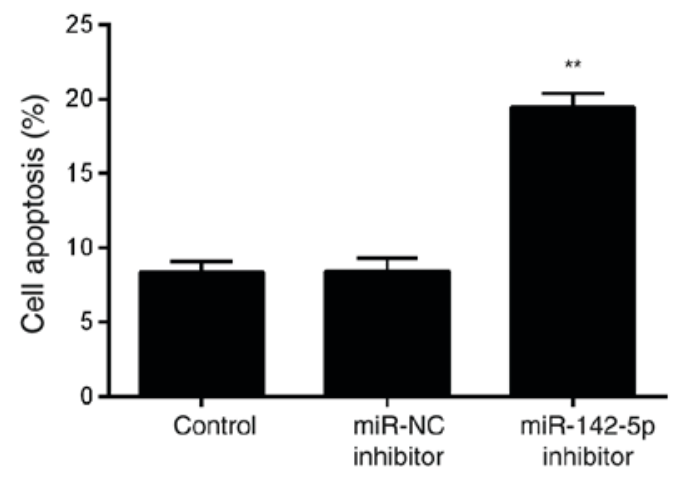

Figure 5. miR-142-5p inhibition increases MDA-MB-231 cell apoptosis. (A) Flow cytometry assay demonstrated that (B) no significant difference was observed between the control and NC groups; inhibition of miR-142-5p significantly induced MDA-MB-231 cell apoptosis. ${ }^{* *} \mathrm{P}<0.01$ vs. control or NC group. miR, microRNA; NC, negative control; FITC, fluorescein isothiocyanate.

A

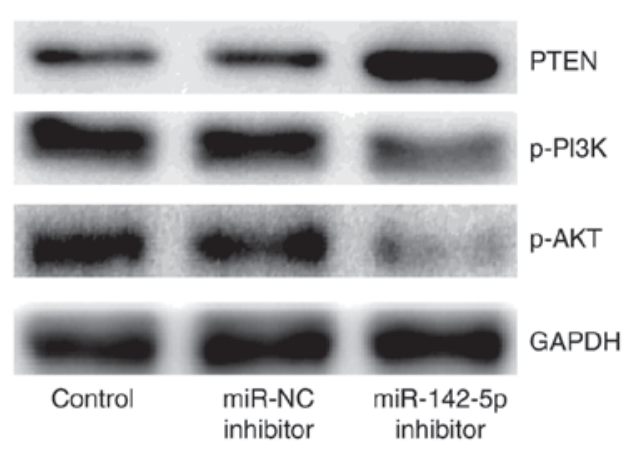

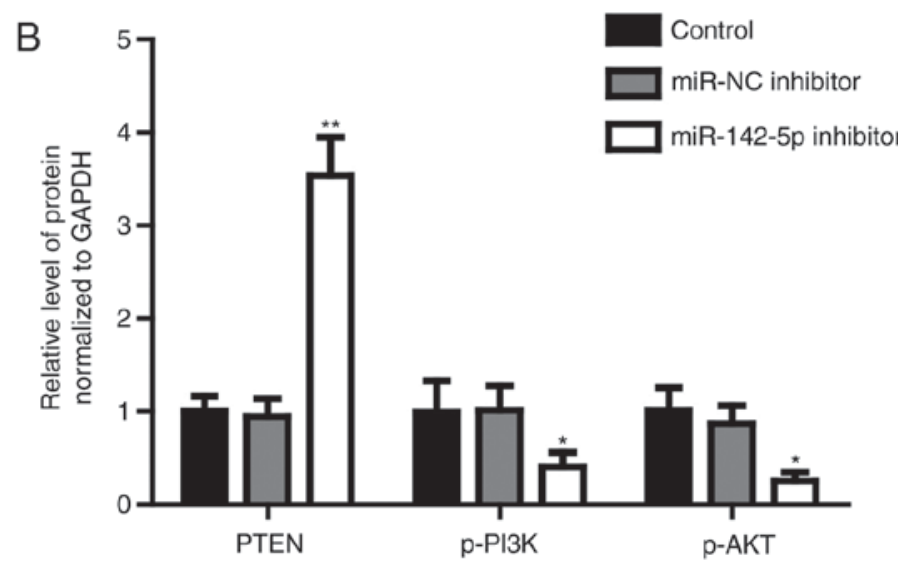

Figure 6. Inhibition of miR-142-5p leads to increased PTEN and reduced p-PI3K and p-AKT protein levels. Results from (A) western blotting and (B) densitometric analysis demonstrated that the inhibition of miR-142-5p resulted in increased PTEN protein expression levels and reduced expression of p-PI3K and p-AKT. ${ }^{\mathrm{P}}<0.05,{ }^{* *} \mathrm{P}<0.01$ vs. Control and miR-NC inhibitor. AKT, RAC $\alpha$ serine/threonine-protein kinase; miR, microRNA; p, phosphorylated; PI3K, phosphatidylinositol-4,5-bisphosphate 3-kinase; PTEN, phosphatase and tensin homolog; NC, negative control.

suggested a potential the interaction between miR-142-5p and PTEN.

Effects of miR-142-5p inhibition on MDA-MB-231 cell viability. MTT assays were conducted to examine the effects of miR-142-5p inhibition on cell viability. No significant differences in viability were identified between Control and miR-NC inhibitor-treated groups; whereas the inhibition of miR-142-5p significantly reduced MDA-MB-231 cell viability at 24 and $48 \mathrm{~h}$ following transfection compared with Control and miR-NC inhibitor groups (Fig. 4).
Effects of miR-142-5p inhibition on MDA-MB-231 cell apoptosis. Flow cytometry results revealed no significant differences between Control and miR-NC inhibitor groups, and that the inhibition of miR-142-5p significantly increased MDA-MB-231 cell apoptosis (Fig. 5).

Effects of miR-142-5p inhibition on PTEN, $p-P I 3 K$ and $p$-AKT protein expression levels. The protein expression levels of PTEN, p-PI3K and p-AKT were examined by western blot analysis, which revealed no significant difference between the Control and miR-NC inhibitor groups; the 
A

Position 2503-2509 of PTEN 3' UTR

hsa-miR-142-5p

Mutant position of PTEN 3' UTR $5^{\prime}$

$3^{\prime}$

$5^{\prime}$
...CGAGUCCUAAUUAAAACUUUAUU...

UCAUCACGAAAGAUGAAAUAC

...CGAGUCCUAAUUAAACCAGAUGU...

B

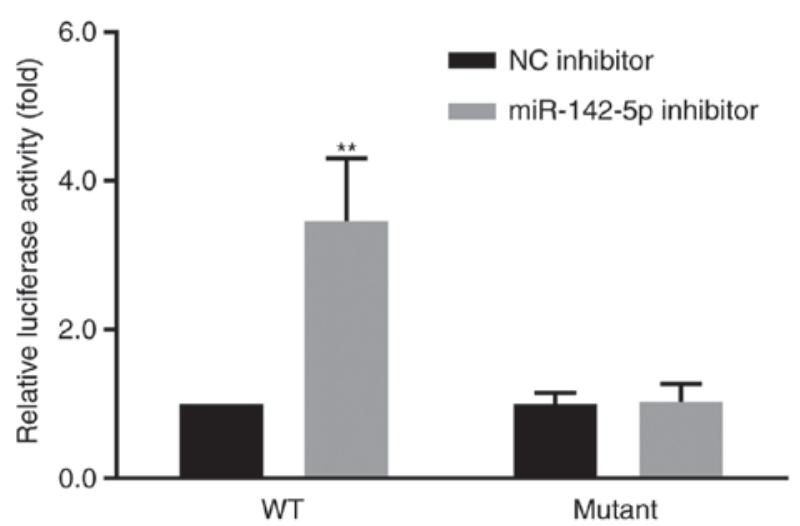

Figure 7. miR-142-5p targets PTEN in MDA-MB-231 cells. (A) The binding site between miR-142-5p and PTEN 3'UTR and the mutant position of PTEN 3'UTR. (B) Similar levels of luciferase activity were observed in the cells harboring the mutant 3'UTR and the WT/NC control cells. Compared with the miR-NC inhibitor, transfection of miR-142-5p inhibitor and PTEN-3'UTR-WT vector but not the PTEN-3'UTR-mutant vector induced the luciferase activity in MDA-MB-231 cells. ${ }^{* *} \mathrm{P}<0.01$ vs. miR-NC inhibitor. miR, microRNA; PTEN, phosphatase and tensin homolog; NC, negative control; UTR, untranslated region; WT, wild type.

inhibition of miR-142-5p significantly increased the expression of PTEN and reduced the expression of p-PI3K and p-AKT in MDA-MB-231 cells compared with the Control and miR-NC inhibitor-treated groups (Fig. 6).

Interaction between miR-142-5p and PTEN. To verify whether PTEN was a direct target of miR-142-5p, a portion of the PTEN 3'UTR containing a putative miR-142-5p target site was cloned into the luciferase open reading frame. Co-transfection of the miR-142-5p inhibitor and the PTEN-3'UTR-WT vector into MDA-MB-231 cells (pLuc-PTEN-WT 3'UTR) exhibited a significant increase in the luciferase activity compared with miR-NC inhibitor. However, no significant difference was identified in luciferase activity in cells co-transfected with the miR-142-5p inhibitor and the mutant PTEN 3'UTR vector (pLuc-PTEN-Mut 3'UTR); similar levels of luciferase activity were observed in the cells harboring the mutant 3'UTR and WT-NC control cells (Fig. 7).

\section{Discussion}

$\mathrm{BC}$ affects $12 \%$ women worldwide (3), thus it is urgent to investigate current diagnostic methods (5). miR-142 was upregulated in BCSCs compared with in non-tumorigenic BC cells (14). Additionally, miR-142-5p expression levels were reported to be higher in patients with $\mathrm{BC}$ with lymph node metastasis compared with patients without lymph node metastasis (15). The present study aimed to investigate a target of miR-142-5p in BC cells and provide a novel target for the treatment of $\mathrm{BC}$.

RT-qPCR revealed that miR-142-5p expression levels were significantly higher in BC tissues compared with adjacent healthy tissues. These data suggested a potential oncogenic role of miR-142-5p in BC, which was consistent with previous studies of BC $(14,15)$. Subsequently, TargetScan was employed to predict the mRNA targets of miR-142-5p, which revealed that the target sequence of miR-142-5p was present in the 3'UTR of PTEN. In addition, RT-qPCR demonstrated that the expression levels of PTEN were lower in BC tissues compared with adjacent healthy tissues, which was in accordance with a previous report that PTEN gene expression levels were lower in 93 human $\mathrm{BC}$ tissues compared with in healthy breast tissues (21). These results suggested that the overexpression of miR-142-5p may inhibit PTEN mRNA expression in BC tissues.

The PTEN protein is a protein tyrosine phosphatase that is encoded by the PTEN gene (22) and may be mutated in human brain, breast and prostate cancers (23). Subsequently, the association of miR-142-5p and PTEN expression with clinicopathological characteristics in patient with BC were investigated. No significant association between miR-142-5p or PTEN expression levels and patient age was observed in present study; however, miR-142-5p and PTEN expression levels were positively and negatively associated, respectively, with patient tumor size and metastasis. The results of the present study suggested that miR-142-5p may have negatively affected the expression levels of PTEN in BC tissues. Additionally, in vitro experiments were conducted to investigate the effect of miR-142-5p expression in human BC cell lines. The MDA-MB-231 BC cell line exhibited a high expression level of miR-142-5p, which was reduced upon transfection with the miR-142-5p inhibitor, whereas mRNA expression levels of PTEN increased. These data further suggested an interaction and a negative association between miR-142-5p and PTEN expression.

Results from the present study demonstrated that inhibition of miR-142-5p significantly reduced MDA-MB-231 cell viability and increased apoptosis. These data were in line with previous studies that have reported an upregulation 
of miR-142-5p expression levels in breast cancer patients with lymph node metastasis compared with those without lymph node metastasis (15), atherosclerotic plaques (16), Hashimoto's thyroiditis (18) and in the serum of patients with colorectal cancer (19), which was associated with the promotion of cell proliferation and colony formation, and with the inhibition of apoptosis in colorectal cancer cell lines by targeting succinate dehydrogenase (ubiquinone) iron-sulfur subunit B (19). miR-142-5p was also reported to be elevated in human renal cell carcinoma tissues, and was demonstrated to induce growth and migration of renal cell carcinoma cells by targeting BTG anti-proliferation factor 3 (24); however, the mechanisms responsible for the alterations in $\mathrm{BC}$ required further investigation. Therefore, western blotting was performed in the present study to analyze protein expression levels of PTEN; in cells transfected with the miR-142-5p inhibitor, a significant increase in the expression of PTEN was observed. These results suggested that the expression of PTEN may be affected by miR-142-5p. The present study also aimed to determine the molecules that may be regulated and influenced by PTEN by using western blot analysis.

The PI3K signaling pathway is an important kinase cascade that regulates cell proliferation, migration, apoptosis and angiogenesis through its downstream effector AKT; dysregulation of this pathway may be caused by mutations or altered expression levels of an upstream regulator of AKT activity such as PTEN $(25,26)$. Previous studies have also reported that mutations in $\mathrm{PI} 3 \mathrm{~K} \alpha$ were common in $\mathrm{BC}$ and the mutation frequency ranged between 27 and 36\% (27). Additionally, PTEN was reported to be a regulator of PI3K cytoplasmic signaling (28) and an inhibitor of the growth-promoting PI3K/AKT/mechanistic target of rapamycin (mTOR) signaling pathway (29). Furthermore, a lack of PTEN protein expression was reported to be associated with overactivation of the PI3K/AKT/mTOR signaling pathway (30). In the present study, western blotting revealed that the inhibition of miR-142-5p significantly reduced the expression of p-PI3K and p-AKT in MDA-MB-231 cells, which agreed with the above studies that further inferred that PI3K or AKT expression was attributed to PTEN.

Results from the luciferase activity assay demonstrated that PTEN was a direct target of miR-142-5p, confirmed by higher luciferase activity after miR-142-5p inhibitor administration in WT group than in the mutant group. Additionally, similar levels of luciferase activity were observed in the cells harboring the WT 3'UTR between the miR-142-5p inhibitor group and miR-NC group in the present study. In a recent study, miR-142-5p was found to target 3'UTR of PTEN in cutaneous squamous cell carcinoma by Bai et al (31), which was in line with the findings of the present study.

In conclusion, miR-142-5p may be a possible target for treating $\mathrm{BC}$ in the future; the PTEN/p-PI3K/p-AKT signaling pathway was associated with the effects of miR-142-5p in MDA-MB-231 cells. However, the effects of miR-142-5p mimics on $\mathrm{BC}$ were not analyzed in the present study and should be investigated in the future; the effects on PI3K or AKT expression attributed to PTEN, can only be inferred, as no direct evidence has been provided. It is necessary for this to be explored in future studies.

\section{Acknowledgements}

Not applicable.

\section{Funding}

No funding was received.

\section{Availability of data and materials}

The datasets used and/or analyzed during the current study are available from the corresponding author on reasonable request.

\section{Authors' contributions}

WX designed and carried out the experiments and analyzed the data. WW designed the experiments, analyzed the data and prepared the manuscript. Both authors have seen and approved the manuscript.

\section{Ethics approval and consent to participate}

The present study was approved by the ethics committee of Renmin Hospital of Wuhan University (Wuhan, China).

\section{Consent for publication}

Written informed consent was obtained from all patients prior to the start of the study.

\section{Competing interests}

The authors declare that they have no competing interests.

\section{References}

1. Siegel RL, Miller KD and Jemal A: Cancer statistics, 2017. CA Cancer J Clin 67: 7-30, 2017.

2. DeSantis C, Siegel R, Bandi P and Jemal A: Breast cancer statistics, 2011. CA Cancer J Clin 61: 409-418, 2011.

3. McGuire A, Brown JA, Malone C, McLaughlin R and Kerin MJ: Effects of age on the detection and management of breast cancer. Cancers (Basel) 7: 908-929, 2015.

4. Veronesi U, Boyle P, Goldhirsch A, Orecchia R and Viale G: Breast cancer. Lancet 365: 1727-1741, 2005.

5. Zhang BN, Cao XC, Chen JY, Chen J, Fu L, Hu XC, Jiang ZF, Li HY, Liao N, Liu DG, et al: Guidelines on the diagnosis and treatment of breast cancer (2011 edition). Gland Surg 1: 39-61, 2011.

6. Farazi TA, Hoell JI, Morozov P and Tuschl T: MicroRNAs in human cancer. Adv Exp Med Biol 774: 1-20, 2013.

7. Nohata N, Hanazawa T, Enokida $H$ and Seki N: microRNA-1/133a and microRNA-206/133b clusters: Dysregulation and functional roles in human cancers. Oncotarget 3: 9-21, 2012.

8. Melo SA and Esteller M: Dysregulation of microRNAs in cancer: Playing with fire. FEBS Lett 585: 2087-2099, 2011.

9. LereboursF,Cizeron-ClairacG,Susini A,VacherS,Mouret-FourmeE, Belichard C, Brain E, Alberini JL, Spyratos F, Lidereau R and Bieche I: miRNA expression profiling of inflammatory breast cancer identifies a 5-miRNA signature predictive of breast tumor aggressiveness. Int J Cancer 133: 1614-1623, 2013.

10. Jiang S, Zhang HW, Lu MH, He XH, Li Y, Gu H, Liu MF and Wang ED: MicroRNA-155 functions as an OncomiR in breast cancer by targeting the suppressor of cytokine signaling 1 gene. Cancer Res 70: 3119-3127, 2010.

11. Lei Z, Xu G, Wang L, Yang H, Liu X, Zhao J and Zhang HT: MiR-142-3p represses TGF- $\beta$-induced growth inhibition through repression of TGF $\beta R 1$ in non-small cell lung cancer. FASEB J 28: 2696-2704, 2014 
12. Shen WW, Zeng Z, Zhu WX and Fu GH: MiR-142-3p functions as a tumor suppressor by targeting CD133, ABCG2, and Lgr5 in colon cancer cells. J Mol Med (Berl) 91: 989-1000, 2013.

13. Chai S, Tong M, Ng KY, Kwan PS, Chan YP, Fung TM, Lee TK, Wong N, Xie D, Yuan YF, et al: Regulatory role of miR-142-3p on the functional hepatic cancer stem cell marker CD133. Oncotarget 5: 5725-5735, 2001.

14. Isobe T, Hisamori S, Hogan DJ, Zabala M, Hendrickson DG, Dalerba P, Cai S, Scheeren F, Kuo AH, Sikandar SS, et al: miR-142 regulates the tumorigenicity of human breast cancer stem cells through the canonical WNT signaling pathway. eLife 3: e01977, 2014

15. Wang B, Li J, Sun M, Sun L and Zhang X: miRNA expression in breast cancer varies with lymph node metastasis and other clinicopathologic features. IUBMB Life 66: 371-377, 2014.

16. Xu R, Bi C, Song J, Wang L, Ge C, Liu X and Zhang M Upregulation of miR-142-5p in atherosclerotic plaques and regulation of oxidized low-density lipoprotein-induced apoptosis in macrophages. Mol Med Rep 11: 3229-3234, 2015.

17. Su YH, Zhou Z, Yang KP, Wang XG, Zhu Y and Fa XE: MIR-142-5p and miR-9 may be involved in squamous lung cancer by regulating cell cycle related genes. Eur Rev Med Pharmacol Sci 17: 3213-3220, 2013.

18. Zhu J, Zhang Y, Zhang W, Zhang W, Fan L, Wang L, Liu Y, Liu S, Guo Y, Wang Y, et al: MicroRNA-142-5p contributes to Hashimoto's thyroiditis by targeting CLDN1. J Transl Med 14: 166, 2016.

19. Liu S, Xiao Z, Ai F, Liu F, Chen X, Cao K, Ren W, Zhang X, Shu P and Zhang D: miR-142-5p promotes development of colorectal cancer through targeting SDHB and facilitating generation of aerobic glycolysis. Biomed Pharmacother 92: 1119-1127, 2017.

20. Livak KJ and Schmittgen TD: Analysis of relative gene expression data using real-time quantitative PCR and the 2(-Delta Delta C(T)) method. Methods 25: 402-408, 2001.

21. Zhang Y, Liu M, Yang H, Wang J, Liu H, Li X, Li J, Xu J and Li X: PIK3CA mutations are a predictor of docetaxel plus epirubicin neoadjuvant chemotherapy clinical efficacy in breast cancer. Neoplasma 61: 461-467, 2014.

22. Song MS, Salmena L and Pandolfi PP: The functions and regulation of the PTEN tumour suppressor. Nat Rev Mol Cell Biol 13: 283-296, 2012.
23. Li J, Yen C, Liaw D, Podsypanina K, Bose S, Wang SI, Puc J, Miliaresis C, Rodgers L, McCombie R, et al: PTEN, a putative protein tyrosine phosphatase gene mutated in human brain, breast, and prostate cancer. Science 275: 1943-1947, 1997.

24. Liu L, Liu S, Duan Q, Chen L, Wu T, Qian H, Yang S, Xin D, He Z and Guo Y: MicroRNA-142-5p promoted cell growth and migration in renal cell carcinoma by targeting BTG3. Am J Transl Res 9: 2394-2402, 2017.

25. Stemke-Hale K, Gonzalez-Angulo AM, Lluch A, Neve RM, Kuo WL, Davies M, Carey M, Hu Z, Guan Y, Sahin A, et al: An integrative genomic and proteomic analysis of PIK3CA, PTEN, and AKT mutations in breast cancer. Cancer Res 68: 6084-6091, 2008.

26. Nagata Y, Lan KH, Zhou X, Tan M, Esteva FJ, Sahin AA, Klos KS, Li P, Monia BP, Nguyen NT, et al: PTEN activation contributes to tumor inhibition by trastuzumab, and loss of PTEN predicts trastuzumab resistance in patients. Cancer Cell 6: 117-127, 2004.

27. Board RE, Thelwell NJ, Ravetto PF, Little S, Ranson M, Dive C, Hughes A and Whitcombe D: Multiplexed assays for detection of mutations in PIK3CA. Clin Chem 54: 757-760, 2008.

28. Milella M, Falcone I, Conciatori F, Cesta Incani U, Del Curatolo A, Inzerilli N, Nuzzo CM, Vaccaro V, Vari S, Cognetti F and Ciuffreda L: PTEN: Multiple functions in human malignant tumors. Front Oncol 5: 24, 2015.

29. Baselga J: Targeting the phosphoinositide-3 (PI3) kinase pathway in breast cancer. Oncologist 16 (Suppl 1): S12-S19, 2011.

30. Carracedo A and Pandolfi PP: The PTEN-PI3K pathway: Of feedbacks and cross-talks. Oncogene 27: 5527-5541, 2008.

31. Bai X, Zhou Y, Chen P, Yang M and Xu J: MicroRNA-142-5p induces cancer stem cell-like properties of cutaneous squamous cell carcinoma via inhibiting PTEN. J Cell Biochem 119: 2179-2188, 2018

This work is licensed under a Creative Commons Attribution-NonCommercial-NoDerivatives 4.0 International (CC BY-NC-ND 4.0) License. 